\title{
Coordinated Control of Traffic Signals for Multiple Intersections
}

\author{
Liqiang Fan \\ Department of Information and Computing Science, Langfang Teachers University, Langfang, China \\ Email: flqabc@126.com
}

Received 7 April 2014; revised 21 May 2014; accepted 1 June 2014

Copyright (C) 2014 by author and Scientific Research Publishing Inc. This work is licensed under the Creative Commons Attribution International License (CC BY). http://creativecommons.org/licenses/by/4.0/

cC) (7) Open Access

\begin{abstract}
The proper phase difference of traffic signals for adjacent intersections could decrease the time of operational delay. Some theorems show how to minimize the total average delay time for vehicle operating at adjacent intersections under given conditions. If the distance and signal cycles of adjacent intersections satisfy with specific conditions, the total average delay time would achieve zero. If the signal cycles of adjacent intersections and the phase difference of them are co-prime numbers, the total average delay time would be a constant. In general, if signal cycles of adjacent intersections and the phase difference of them are reducible numbers, the minimum total average delay time would be solved by the given algorithm. Numerical experiments have verified the rationality of these theorems.
\end{abstract}

\section{Keywords}

\section{Coordinated Control, Adjacent Intersections, Phase Difference, Average Delay Time}

\section{Introduction}

With the increase of urban traffic demand, urban traffic problems become more and more serious. The technology of traffic signal coordinated control plays an important role in easing traffic congestion of cities [1]. The purpose of traffic signal coordinated control is to design a method to minimize the operational delay of vehicles. Up to now, many models have been constructed in terms of traffic signal coordinated control [2] [3].

Arterial roads load the main traffic flow of the whole city. To install traffic signals at adjacent intersections could decrease the time for operational delay [4]. In the traffic signal coordinated control system of arterial roads, the traffic signals could coordinate under the condition of the same cycles for all of them [5] [6]. Therefore, the traffic signal coordinated control system of arterial roads has obvious disadvantages, namely, such traffic signal coordinated control system could not apply to all intersections. As a result, this condition hinders the 
development of the technology of traffic signal coordinated control [7].

For traffic signal coordinated control of intersections of different signal cycles, related models have been built [8] [9]. These models need to fix the cycle of traffic signals at one intersection firstly, and then give the cycle and phase difference of adjacent intersections to minimize the total average operational delay. In fact, cycles of signals at intersections are determined by the actual traffic flow on the roads and cycles of traffic signals at intersections do not depend on adjacent intersections. So the control method of phase difference of traffic signals is considered under the condition that the cycles of signals at intersections have been fully given. To be specific, the total average delay time of vehicles on given roads is minimized by choosing proper phase difference. As the technology of traffic signal coordinated control is mainly aimed at easing the traffic congestion, this article takes account of only the traffic signal coordinated control under the condition of approximately saturated traffic flows and obvious vehicle-following phenomenon.

\section{Auxiliary Theorem}

In order to give the minimum average delay time for vehicle operating at adjacent intersections, we give the following periodic sequence and preparatory theorem firstly.

Suppose that $a, b, c, d, \Delta x$ are positive integers and satisfy the finite conditions of $a=b+c$ and $b>\max \{c, d, \Delta x\}$. Sequence $T^{*}$ could be given in the following form.

$$
\left\{\begin{array} { l } 
{ d , d - \Delta x , \cdots , d - [ \frac { d } { \Delta x } ] \Delta x = p _ { 1 } , } \\
{ \frac { b } { c } ( \Delta x - p _ { 1 } ) , \frac { b } { c } ( 2 \Delta x - p _ { 1 } ) , \cdots , \frac { b } { c } q _ { 1 } , }
\end{array} \text { and } \left\{\begin{array}{l}
b+\left(c-q_{i-1}\right)-\Delta x, b+\left(c-q_{i-1}\right)-2 \Delta x, \cdots, p_{i}, \\
\frac{b}{c}\left(\Delta x-p_{i}\right), \frac{b}{c}\left(2 \Delta x-p_{i}\right), \cdots, \frac{b}{c} q_{i},
\end{array}\right.\right.
$$

$i=2,3, \cdots$, where $q_{1}=\left[\frac{c+p_{1}}{\Delta x}\right] \Delta x-p_{1}, \quad p_{i}=b+\left(c-q_{i-1}\right)-\Delta x-\left[\frac{b+\left(c-q_{i-1}\right)-\Delta x}{\Delta x}\right] \Delta x$, and

$q_{i}=\left[\frac{c+p_{i+1}}{\Delta x}\right] \Delta x-p_{i},[\cdot]$ denotes the rounding operation.

Theorem 1: $T^{*}$ which is constructed above is a periodic sequence, and $\frac{e}{\Delta x}$ is the minimal positive cycle, where $e$ is the smallest common multiple of $a$ and $\Delta x$.

Proof. Let $b, c$ be positive integers, and set $A=\left\{b, b-1, \cdots, 1,0,1 \cdot \frac{b}{c}, 2 \cdot \frac{b}{c}, \cdots,(c-1) \cdot \frac{b}{c}\right\}$.

Then, the number of elements in set $A$ is $b+c$.

List the elements in set $A$ into the following Figure 1.

Elements in Sequence $T^{*}$ are gained by beginning from element $d$ on the circular disc, selecting one clockwise from the circular disc every other $\Delta x-1$ elements. Since $e$ is the smallest common multiple of $a$ and $\Delta x$, the difference of $\frac{e}{\Delta x}$ and $d$ in $T^{*}$ is 0 . In other words, starting from element $d$ on the circular disc, it may return to the starting point after $\Delta x$ circles.

This completes the proof.

\section{The Minimum Total Average Delay Time of Vehicles}

\subsection{Basic Assumptions}

For the convenience of narrating, geometric figure applies 4-phases control regarding the traffic lights at intersections. The related four phases are given in Figure 2.

According to the characteristics of urban traffic flows, in order to give the best design for traffic signals, the following assumptions are given for traffic flows in this section.

1) The vehicles keep driving at uniform speed on fixed roads (the speed is different on different roads). The cycles of traffic signals at every intersection are reasonable (the vehicles do not overflow) with the duration of 


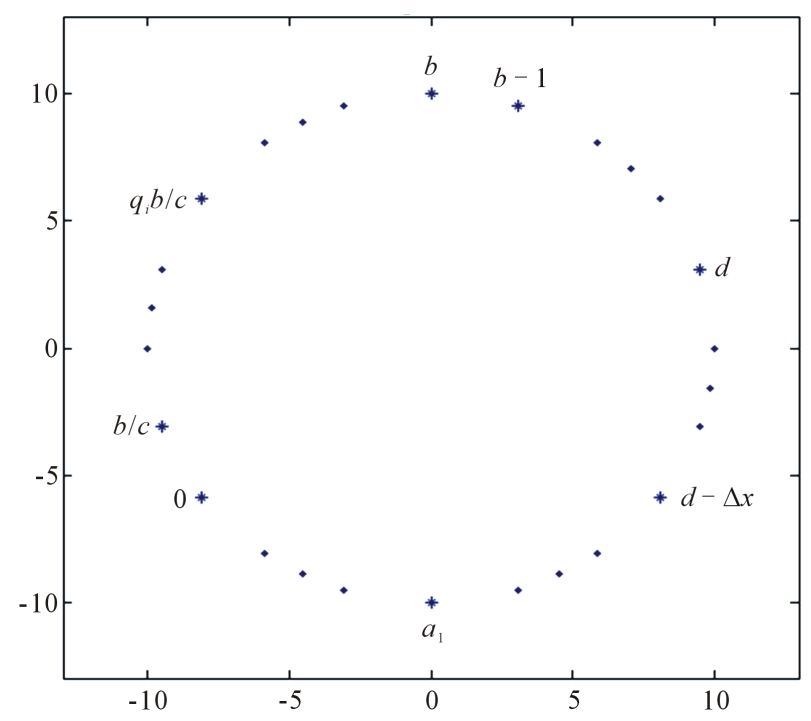

Figure 1. Elements in set $A$.

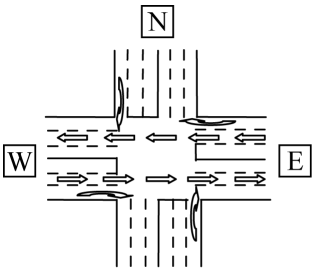

$\mathrm{S}$

The first phase

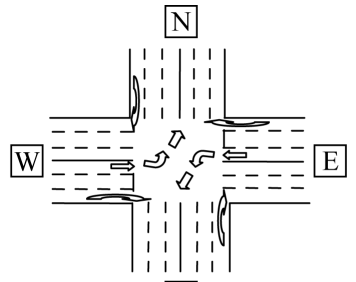

$\mathrm{S}$

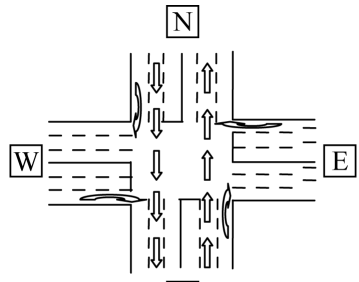

S

The second phase

The third phase

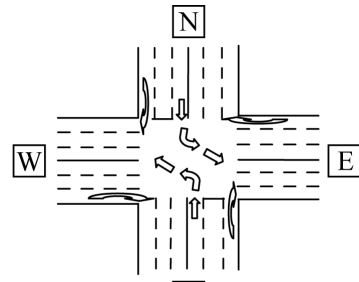

$\mathrm{S}$

The fourth phase

Figure 2. Four phases in an ordinary four way intersections.

yellow light ignored;

2) The traffic flows are stable and become saturated within the corresponding phase;

3) The traffic flows in straight direction (left-turn direction) and right-turn direction have been in conflux before they arrive at the next intersection;

4) The cycle of Intersection $A$ is $T_{A}$, the first phase period of Intersection $A$ is $t_{A}$, the cycle of Intersection $B$ is $T_{B}$ and the first phase period of Intersection $B$ is $t_{B} . S$ is the smallest common multiple of $T_{A}$ and $T_{B}$, $n_{A}=S / T_{A}$ and $n_{B}=S / T_{B}$,

5) The travel time at Intersection A and Intersection B is $t_{x}$.

\subsection{The Minimum Total Average Delay Time}

In general, when we drive from one place to another, the times of turning left or right continuously will not exceed three, otherwise, the travelling route will certainly not be optimal. Therefore, we focus on the newest average delay time of vehicles in straight direction in this article.

Theorem 2. Suppose that the cycles of signals for Intersection A and B are $T_{A}$ and $T_{B}$ and the travel time from Intersection A to B is $t_{x}$. If $T_{A}=T_{B}=T$ and $t_{x}$ is divided exactly by $T / 2$, then proper control method can be chosen to enable the minimum total average delay time to be 0 .

Proof. The time ranges for the traffic flows to arrive at Intersection A are

$$
\left[0, t_{A}\right],\left[T, t_{A}+T\right], \cdots,\left[\left(n_{A}-1\right) T, t_{A}+\left(n_{A}-1\right) T\right], \cdots .
$$

When these traffic flows leave Intersection A, the confluence and diffluence of vehicles have been completed before they arrive at Intersection B. And when they arrive at Intersection B, the time ranges of new traffic flows are 


$$
\left[t_{x}, t_{x}+t_{B}\right],\left[t_{x}+T, t_{x}+t_{B}+T\right], \cdots,\left[t_{x}+\left(n_{A}-1\right) T, t_{x}+t_{B}+\left(n_{A}-1\right) T\right], \cdots .
$$

If $\Delta t=t_{x}-\omega T_{B}>0$, where $\omega$ is a nonnegative integer, the time ranges of first phase of traffic signals at Intersection $\mathrm{B}$ are

$$
\left[t_{x}-\omega T, t_{B}+t_{x}-\omega T\right],\left[t_{x}-(\omega-1) T, t_{B}+t_{x}-(\omega-1) T\right], \cdots,\left[t_{x}+\left(n_{B}-\omega-1\right) T, t_{x}+t_{B}+\left(n_{B}-\omega-1\right) T\right], \cdots
$$

From this it can be known that the average waiting time is $W_{B}=0$ at this moment.

Similarly, in the direction of from Intersection B to Intersection A, when the traffic flows leave Intersection B, the confluence and diffluence of vehicles have been completed before they arrive at Intersection A. The time ranges for the new traffic flows to arrive at Intersection A are

$$
\begin{aligned}
& {\left[2 t_{x}-\omega T, t_{A}+2 t_{x}-\omega T\right],\left[2 t_{x}-(\omega-1) T, t_{A}+2 t_{x}-(\omega-1) T\right], \cdots,} \\
& {\left[2 t_{x}+\left(n_{B}-\omega-1\right) T, 2 t_{x}+t_{A}+\left(n_{B}-\omega-1\right) T\right], \cdots .}
\end{aligned}
$$

Since $2 t_{x}$ can be divided exactly by $T$, the total average delay time is $W_{A}=0$.

From the above, the total average delay time is minimal, with the minimum of 0 .

Theorem 3. Suppose that the cycles of signals at Intersection $A$ and $B$ are $T_{A}$ and $T_{B}$ and the travel time for driving from Intersection $\mathrm{A}$ to $\mathrm{B}$ is $t_{x}$. If $T_{A}$ and $\Delta T=\left|T_{A}-T_{B}\right|$ are co-prime numbers and $T_{B}$ and $\Delta T$ are co-prime numbers, then the minimum total average delay time $W_{A}+W_{B}=\frac{T_{A}\left(T_{A}-t_{A}\right)}{2}+\frac{T_{B}\left(T_{B}-t_{B}\right)}{2}$ is a constant.

Proof. We suppose that the first vehicle drives through Intersection A at time 0 and when it arrives at Intersection $\mathrm{B}$, this vehicle (in through lane) would wait for $d$ seconds before it leaves Intersection $\mathrm{B}$.

Let $T_{B}=a, \Delta T=\Delta x, b=T_{B}-t_{B}$ and $c=t_{B}$. If the time ranges of traffic flows at Intersection $A$ are

$$
\left[0, t_{A}\right],\left[T_{A}, t_{A}+T_{A}\right], \cdots,\left[\left(n_{A}-1\right) T_{A}, t_{A}+\left(n_{A}-1\right) T_{A}\right], \cdots,
$$

then the elements of periodic sequence $T^{*}$ are the delay time of traffic flows stopping at Intersection $B$.

Since $T_{B}$ and $\Delta T$ are relatively prime, the smallest common multiple of $T_{B}$ and $\Delta T$ is $T_{B} \Delta T$ and the number of different elements in $T^{*}$ is $T_{B}$. Thus the total average delay time $W_{B}=\frac{T_{B}\left(T_{B}-t_{B}\right)}{2}$ in period $T_{A} T_{B}$.

Similarly, $W_{A}=\frac{T_{A}\left(T_{A}-t_{A}\right)}{2}$. This completes the proof.

Particularly, we note that $W_{A}+W_{B}$ does not rely on the value of $d$ and $t_{x}$ under the condition of Theorem 3 .

Theorem 4. Suppose that the cycles of signals at Intersection $A$ and $B$ are $T_{A}$ and $T_{B}$, and the travel time for driving from Intersection A to B is $t_{x}$. If $T_{A} \neq T_{B}, S_{A}$ is the smallest common multiple of $T_{A}$ and $\Delta T=\left|T_{A}-T_{B}\right|$, and $S_{B}$ is the smallest common multiple of $T_{B}$ and $\Delta T$, then there is a coordinated control method which could minimize the total average delay time $W_{A}+W_{B}$ in the time ranges of $S=T_{A} T_{B}$.

Proof. We suppose that the first vehicle drives through Intersection $A$ at time 0 , and when it arrives at Intersection $\mathrm{B}$, this vehicle (in through lane) would wait for $d$ seconds before it leaves Intersection $\mathrm{B}$.

Let $T_{B}=a, \Delta T=\Delta x, b=T_{B}-t_{B}$ and $c=t_{B}$. Let the time ranges of traffic flows at Intersection $A$ to be

$$
\left[0, t_{A}\right],\left[T_{A}, t_{A}+T_{A}\right], \cdots,\left[\left(n_{A}-1\right) T_{A}, t_{A}+\left(n_{A}-1\right) T_{A}\right], \cdots .
$$

Then, the elements of periodic sequence $T_{B}^{*}$ (the constructing process similar to $T^{*}$ ) are the delay time of traffic flows that drive from Intersection A to Intersection B.

Since $S_{B}$ is the smallest common multiple of $T_{B}$ and $\Delta T=\left|T_{A}-T_{B}\right|$, by Theorem 1 , we know that the number of $T_{B}^{*}$ is $S_{B} / \Delta T$ and $W_{B}$ is the sum of every element in $T_{B}^{*} . W_{B}$ would change by the different $d$ (the average delay time of the first traffic flow which drive from Intersection $\mathrm{A}$ ).

Similarly, the changes of $W_{A}$ also depend on the different $d$.

Since $d \in A$ and the number of set $A$ is finite, the number of the different $W_{A}+W_{B}$ is finite, we can select the minimum one from these finite elements. 
The proof is completed.

Note that Theorem 3 is a special case of Theorem 4 .

\subsection{The Algorithm of Searching for the Minimum Total Average Delay Time}

Theorem 4 shows the existence of the minimum total average delay only. Now we give the algorithm of searching for minimum total average delay time.

Step 1. Compute $S_{A}$ (the smallest common multiple of $T_{A}$ and $\Delta T$ ), $S_{B}$ and $S$. Let the phase difference $\Delta t=0$;

Step 2. Let

$$
d_{A}= \begin{cases}t_{A}-r\left(\Delta t+t_{\chi}, T_{A}\right), & r\left(\Delta t+t_{x}, T_{A}\right) \geq t_{A}, \\ \frac{r\left(\Delta t+t_{\chi}, T_{A}\right)}{t_{A}}\left(T_{A}-t_{A}\right), & r\left(\Delta t+t_{\chi}, T_{A}\right)<t_{A},\end{cases}
$$

and

$$
d_{B}= \begin{cases}\Delta t-r\left(t_{x}, T_{B}\right), & r\left(t_{x}, T_{B}\right) \leq \Delta t, \\ \frac{r\left(t_{x}, T_{B}\right)-\Delta t}{t_{B}}\left(T_{B}-t_{B}\right), & \Delta t<r\left(t_{x}, T_{B}\right) \leq \Delta t+t_{B}, \\ \Delta t+T_{B}-r\left(t_{x}, T_{B}\right), & r\left(t_{x}, T_{B}\right)>\Delta t+t_{B},\end{cases}
$$

where $r\left(t_{x}, T_{B}\right)=t_{x}-\left[\frac{t_{x}}{T_{B}}\right] T_{B}$;

Step 3. Construct sequence $T_{B}^{*}$ and $T_{A}^{*}$. The elements of $T_{A}^{*}$ are the series beginning from $d_{A}$, and choosing one element from the set $\left\{0,1,2, \cdots, T_{A}-t_{A}, \frac{t_{A}-1}{t_{A}}\left(T_{A}-t_{A}\right), \cdots, \frac{1}{t_{A}}\left(T_{A}-t_{A}\right)\right\}$ (refer to Figure 1) after $\Delta T-1$ elements in counter clockwise direction;

Step 4. Compute $W_{A}$ (the sum of all elements in $T_{A}^{*}$ ), $W_{B}$ and $W=\frac{T_{A} \Delta T}{s_{A}} W_{A}+\frac{T_{B} \Delta T}{s_{B}} W_{B}$;

Step 5. If $\Delta t \neq T_{B}-1$, let $\Delta t=\Delta t+1$, go to Step 2;

Step 6. Select the minimal $W$ and then the corresponding $\Delta t$ is the optimum phase difference.

Note that the algorithm can be optimized by Theorem 2 and Theorem 3 .

\subsection{The Total Average Delay Time for Multiple Intersections}

Further, for the traffic network shown by Figure 3, we can first set the beginning time of the first phase of Intersection C, and then give the beginning time of Intersection B according to the method as provided in Section 3.2, and give the beginning time of the second phases of Intersection $\mathrm{D}$ and $\mathrm{E}$ by means of the beginning time of the second phase of Intersection B till the beginning time of signals at all intersections in the road network is given.

\section{Experiments}

In this section, we give some examples.

Example 1. Let the cycles of Intersection A and B are $T_{A}=91(\mathrm{~s}), T_{B}=87(\mathrm{~s})$, the first phase are $t_{A}=30(\mathrm{~s})$, $t_{B}=25(\mathrm{~s})$, and the travel time between Intersection A and Intersection B is $t_{x}=100(\mathrm{~s})$. Let $\Delta t=0,1, \cdots, 86$, the minimum total average delay $W=W^{*}=5.473 \times 10^{3}(\mathrm{~s})$ could be given by the algorithm (section 3.3). By Theorem 3, $W=W_{A}+W_{B}=\frac{T_{A}\left(T_{A}-t_{A}\right)}{2}+\frac{T_{B}\left(T_{B}-t_{B}\right)}{2}=5.473 \times 10^{3}$ in period $T_{A} T_{B}=7917(\mathrm{~s})$.

Figure 4 shows that $W$ does not change with the different phase difference. This result is consistent with Theorem 3. 

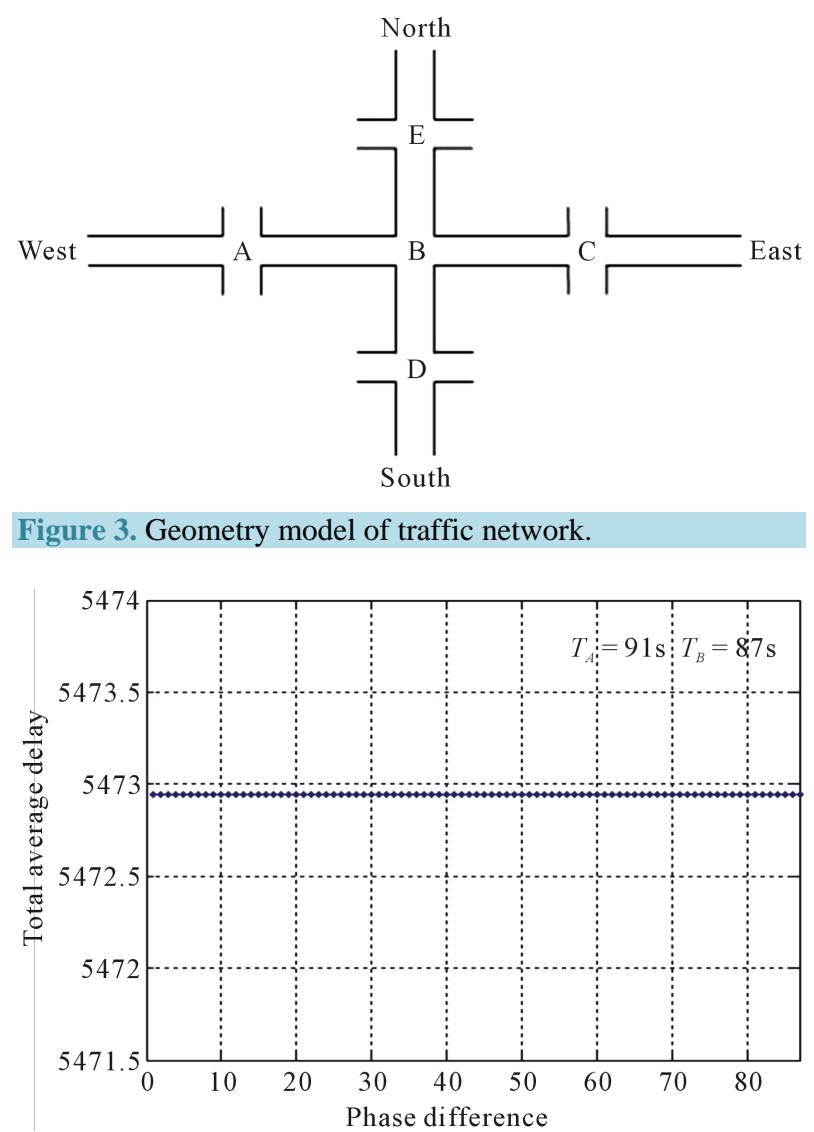

Figure 4. Result of example 1.

Example 2. Let the cycles of Intersection $A$ and $B$ are $T_{A}=90(\mathrm{~s}), T_{B}=80(\mathrm{~s})$, the first phases are $t_{A}=30(\mathrm{~s}), t_{B}=25(\mathrm{~s})$, and the travel time between Intersection A and Intersection B is $t_{x}=100(\mathrm{~s})$. If the phase difference $\Delta t=24(\mathrm{~s})$ and the first phase starts at time 0 , then we can give the following table,

From Table 1 we find that the average delay time appear repeatedly after fixed numbers.

Let $\Delta t=0,1, \cdots, 79$, then the minimum total average delay time $W^{*}=4102(\mathrm{~s})$ could be given by the algorithm (section 3.3), the corresponding phase difference $\Delta t=11(21,41,51,61,71)$ (the period of Intersection $A$ and $B$ is 7200 s).

Figure 5 shows the existence of minimum total average delay time, and this result is consistent with Theorem 4.

Note that the optimal phase difference is not unique.

Example 3. Let the cycles of Intersection $A$ and $B$ are $T_{A}=92(\mathrm{~s}), T_{B}=86(\mathrm{~s})$, the first phases are $t_{A}=30(\mathrm{~s}), t_{B}=25(\mathrm{~s})$, and the travel time between Intersection A and B is $t_{x}=100(\mathrm{~s})$. We suppose that the first phase start at time 0 .

If $\Delta t=0,1, \cdots, 85$, then the minimum total average delay time is $W^{*}=4.732 \times 10^{3}$ which could be given by the algorithm (section 3.3), and the corresponding phase difference is $\Delta t=53$ (the period of Intersection A and $\mathrm{B}$ is $7912 \mathrm{~s})$.

Through the comparison of Figure 4 and Figure 6, we find that the minimum total average delay time would decrease if $T_{A}$ and $\Delta T=\left|T_{A}-T_{B}\right|$ are not co-prime numbers ( $T_{B}$ and $\Delta T$ are not co-prime numbers).

\section{Conclusion}

Under the premise of the vehicles driving at fixed speed on the given roads and some other reasonable assumptions, the vehicles driving on the roads could achieve minimum total average operational delay by setting phase difference of traffic signals at adjacent intersections. This thesis gives the minimum average delay time under 
Table 1. Time ranges of traffic flow.

\begin{tabular}{|c|c|c|c|c|c|}
\hline $\begin{array}{c}\text { Total average delay } \\
\text { time of A }\end{array}$ & $\begin{array}{l}\text { Period of the first } \\
\text { phase of A }\end{array}$ & $\begin{array}{l}\text { Traffic flow arrive } \\
\text { at A from B }\end{array}$ & $\begin{array}{l}\text { Traffic flow arrive } \\
\text { at B from A }\end{array}$ & $\begin{array}{l}\text { Period of the first } \\
\text { phase of B }\end{array}$ & $\begin{array}{c}\text { Total average delay } \\
\text { time of } B\end{array}$ \\
\hline & {$[0,30]$} & & & {$[24,49]$} & \\
\hline & {$[90,120]$} & & {$[100,125]$} & {$[104,129]$} & $4^{*}$ \\
\hline $56,24(90-30) / 30^{*}$ & {$[180,210]$} & {$[124,154],[204,234]$} & {$[190,215]$} & {$[184,209]$} & $6(80-25) / 25$ \\
\hline $14(90-30) / 30$ & {$[270,300]$} & {$[284,314]$} & {$[280,305]$} & {$[264,289]$} & $16(80-25) / 25$ \\
\hline $4(90-30) / 30$ & {$[360,390]$} & {$[364,394]$} & & {$[344,369]$} & \\
\hline 6 & {$[450,480]$} & {$[444,474]$} & {$[370,395]$} & {$[424,449]$} & 54 \\
\hline 16 & {$[540,570]$} & {$[524,554]$} & {$[460,485]$} & {$[504,529]$} & 44 \\
\hline 26 & {$[630,660]$} & {$[604,634]$} & {$[550,575]$} & {$[584,609]$} & 34 \\
\hline 36 & {$[720,750]$} & {$[684,714]$} & {$[640,675]$} & {$[664,689]$} & 24 \\
\hline 46 & {$[810,840]$} & {$[764,794]$} & {$[730,755]$} & {$[744,769]$} & 14 \\
\hline $56,24(90-30) / 30^{*}$ & {$[900,930]$} & {$[844,874],[924,954]$} & {$[820,845]$} & {$[824,849]$} & $4^{*}$ \\
\hline
\end{tabular}

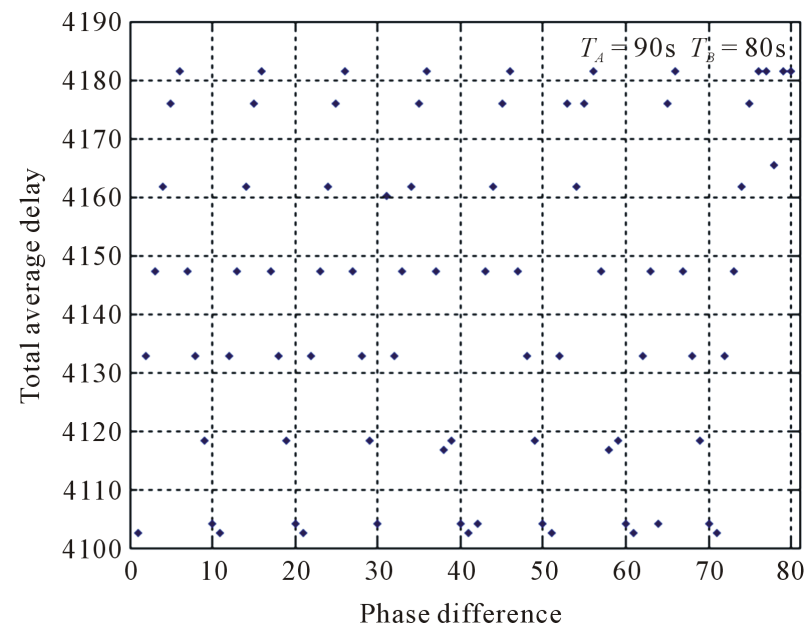

Figure 5. Result of example 2.

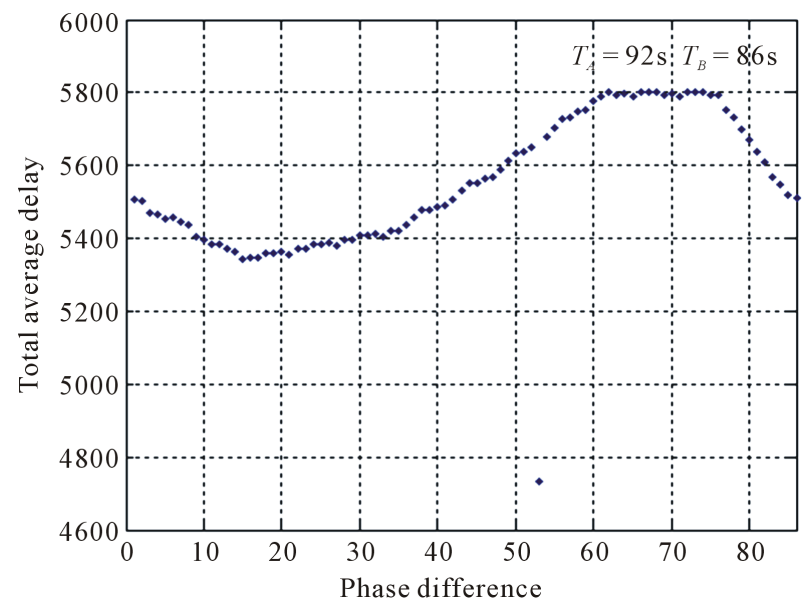

Figure 6. Result of example 3. 
the condition that the cycles of traffic signals meet different finite conditions. In particular, if the cycles of signals at adjacent intersections are co-prime numbers, no matter which value is chosen for phase difference, the minimum average delay is a constant. The three numerical examples have illustrated the rationality of these theorems. The reasonable phase difference of adjacent intersections wherein can be directly computed by the algorithm given in the article

\section{References}

[1] Newell, G. (1998) The Rolling Horizon Scheme of Traffic Signal Control. Transportation Research Part A, 32, 39-44. http://dx.doi.org/10.1016/S0965-8564(97)00017-7

[2] Ji, L. and Song, Q. (2011) Biderectional Green Wave Coordinate Control for Arterial Road under Asymmetric Signal Mode. Journal of Highway and Transportation Research and Development, 28, 96-101.

[3] Simlai, P.E. (2013) Optimum Property of Estimating Function for Spatial Auto-Regressive Models. International Journal of Statistics and Economics, 11, 1-13.

[4] Zang, L. and Lei, J. (2007) Study on Dynamic and Cooperative Control for Neighboring Intersection on Traffic Arterial Roads. Journal of Highway and Transportation Research and Development, 24, 103-106.

[5] Mou, H. and Yu, J. (2011) Traffic Signal Control of Urban Traffic Intersection Group Based on Fuzzy Control. Journal of Lanzhou Jiao Tong University, 30, 106-110.

[6] Liang, J. and Xu, J. (2011) A Coordinated Control Method of Multiple Intersections in Different Cycles. Journal of Highway and Transportation Research and Development, 30, 118-122.

[7] Gartner, N. and Stamatisdis, C. (2002) Arterial-Base Control of Traffic Flow in Urban Grid Networks. Mathematical and Computer Modeling, 35, 657-671. http://dx.doi.org/10.1016/S0895-7177(02)80027-9

[8] Vrcan, Z., Siminiati, D. and Lovrin, N. (2011) Design Proposal for a Hydrostatic City Bus Transmission. Engineering Review, 31, 81-89.

[9] Yeshkeev, A.R. (2013) The Properties of Positive Jonsson's Theories and Their Models. International Journal of Mathematics and Computation, 22, 161-171. 
Scientific Research Publishing (SCIRP) is one of the largest Open Access journal publishers. It is currently publishing more than 200 open access, online, peer-reviewed journals covering a wide range of academic disciplines. SCIRP serves the worldwide academic communities and contributes to the progress and application of science with its publication.

Other selected journals from SCIRP are listed as below. Submit your manuscript to us via either submit@scirp.org or Online Submission Portal.
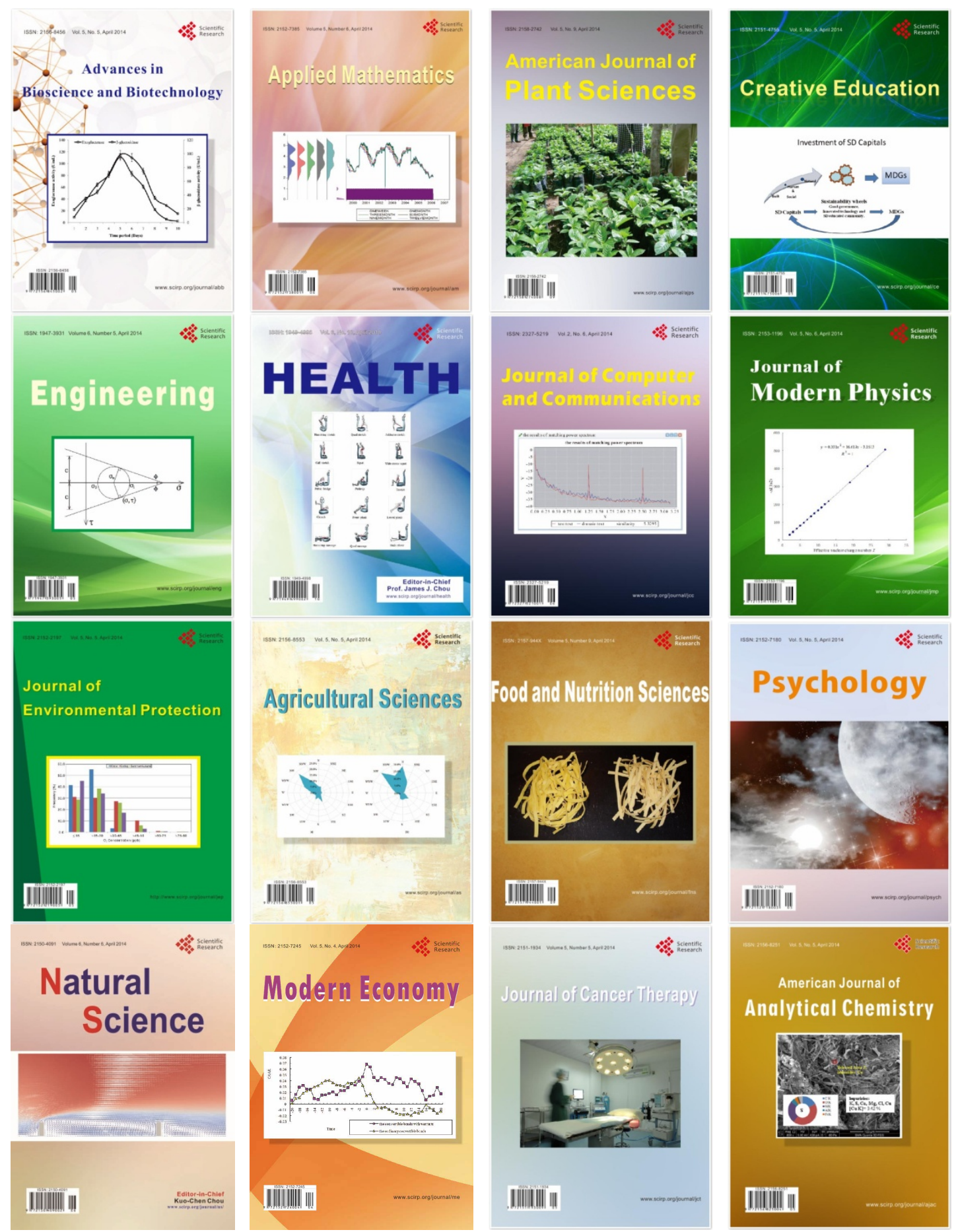